\title{
DEM Reconstruction Based on Adaptive Local RBF
}

\author{
Ping Duan, Yehua Sheng*, Siyang Zhang, Haiyang Lv, and Jia Li
}

Key Laboratory of Virtual Geographic Environment, Ministry of Education, Nanjing Normal University, Nanjing China, 210023, China

\begin{abstract}
As the core of digital elevation model, interpolation methods have been run through the each link, such as production, quality control, accuracy assessment, analytical applications and etc. The local radial basis function interpolation method based on spatial relationship of natural neighbor was proposed in this paper. The interpolation reference points were chosen by the Delaunay Triangulation. The first-order and second-order neighboring of interpolation points as the interpolation reference points were used to construct local radial basis function. This method was applied to the construction of digital elevation model, and the correspondent errors were analyzed. Experimental result shows that the method has a good effect on the construction of different landform.
\end{abstract}

Keywords: Adaptive, delaunay, DEM, error, interpolation, natural neighbor, radial basis function, triangulation.

\section{INTRODUCTION}

Digital Elevation Model (DEM) is the digital representation of the earth's surface. Patent US6748121, titled "Intelligent interpolation methods for automatic generation of an accurate digital elevation model" [1], provided some interpolation techniques to automatically generate DEM and to accurately extract 3D digital elevation models using satellite imagery, aerial photography and cadastral survey data. Patent CN101950436, titled "Method for manufacturing digital elevation model by using data of laser altimeter" [2], provided a technique for generate a DEM by using LiDAR data. Patent CN102339478, titled "Method and device for generating DEM from contour map" [3], disclosed a technique and device for manufacturing a DEM using a contour map as a data source. Patent CN102436679, titled "Medium-resolution remote sensing image discrete point DEM construction method based on medium value filtering" [4], provided a technique for generate a DEM using medium value filtering with medium resolution remote sensing image discrete points. Patent WO2013121340, titled "Digital elevation model" [5], disclosed a technique for enhancing a DEM resolution which improved the precision of the original DEM by using contour map data. The intermediate DEM interpolated from contour map data is fused with the original DEM to generate a higher resolution than the native resolution of the original DEM.

Patents above can improve the accuracy of DEM by mean of various data sources. But, under the condition of the same data source, it is a mathematical problem that how to generate DEM through unevenly distributed sampling points

*Address correspondence to the author at the Yehua Sheng is with Key Laboratory of Virtual Geographic Environment of Ministry of Education, NanJing Normal University, NanJing, China, 210023;

Tel: 0086-025-85891879; E-mail: shengyehua@njnu.edu.cn
[6]. To this problem, many interpolation methods were proposed, like linear interpolation, inverse distance weighted, bivariate spline function, least-squares collocation, natural neighbor, triangular interpolation, Kriging and Radial Basis Function (RBF). Franke analyzed through the reference points, choosing of weighting function, continuity of interpolation function and error statistics, the results show that RBF interpolation method has better reliability than others [7].

With advantages of simple form and convenience of solving, RBF interpolation is widely used in geological prospecting, surface topography, heavy metal pollutant and DEM. Patent US 7024279, titled "Interpolation using radial basis functions with application to inverse kinematics" [8], provided a technique for solving an inverse-kinematic problem by interpolating solutions from examples. Patent WO2012037317, titled "Method and apparatus for predicting petrophysical properties from nmr data in carbonate rocks" [9], disclosed a method to solve a problem of an inverse kinematic problem by interpolating solutions from sampling points.

RBF interpolation is classified into global RBF interpolation and local RBF interpolation. All sampling points are used to construct Global RBF and calculate properties of the interpolation point. However, when there are too many reference points, some problems will occur and will cause the failure of the interpolation, which are singular matrix or the matrix being too large to be solved $[10,11]$. Local RBF interpolation can solve the problems caused by the global RBF search strategy by choosing part of all sample points near the interpolation point to construct RBF. The distance search strategy is usually used to set the range of local RBF, which actually a circle with interpolationpoint as the center and a specific circle radius. The points located in the circle will be chosen as the reference points. However, the radius is not fixed. The radius directly affects the number 
of reference points, too many or too few of which can cause different disadvantages [12].

The spatial relationship of natural neighbor is one of the most important spatial relationships. With good adaptive characteristics about choosing reference points it can effectively solve the problem of reference points' uneven distribution. Through the spatial relationship of natural neighbor, first order and second order neighboring are chosen as the reference points. By this way, reference points are evenly adoptively distributed around interpolationpoint. On this basis, the interpolation calculation is called Adaptive Local RBF (ALRBF). The natural neighbor reference points are usually chosen through Voronoi, but construction of Voronoi is quite complicated and time-consuming. Delaunay is the dual graph of Voronoi. Most importantly, Delaunay has the features of flexibility and accessibility. For any interpolation point, the first order and second order natural neighboring can be adaptively chosen for ALRBF interpolation by partially updating the Delaunay.

Different sections of the thesis are organized as follows. Section 2 describes the detail of ALRBF interpolation including adaptively choosing reference points and RBF. Section 3 analyzes the results of experiments. The content of section 4 is conclusion.

\section{ALRBF INTERPOLATION}

The process of ALRBF goes as follows. Firstly the Minimum Bounding Rectangle (MBR) of point set $S$ is initialized. The interpolation points are generated by divided equidistantly. Construct Delaunay Triangulation for $S$, which is $\mathrm{DT}_{S}$. Insert interpolation points into $\mathrm{DT}_{S}$, and $\mathrm{DT}_{S}$ will be partially updated. Choosing first order and second order natural neighboring as reference points centering on the interpolation point. At last, calculate the interpolation using RBF. Maximum error, minimum error, mean error and rootmean-square error are used to analyze the error of ALRBF.

\subsection{Adaptively choose reference points}

The most vital step of ALRBF is how to adaptively choose reference points. Presumably, construct $\mathrm{DT}_{S}$ based on
$S$, Delaunay needs to search all triangles when inserted a new point $p$. The time and space efficiency is lower and the range of search is larger. In this paper, using the method of the grid index can reduce the time which is taken to search the triangle. The MBR of all the points in data domain is divided into regular grid units. Each triangle has a MBR. If the MBR of the triangle as an approximate body intersects with the regular grid units, the regular grid units can record the triangle index. As shown in Fig. (1), if the point $p$ is included in the triangle $t$, the point $p$ is also included in the MBR of the triangle $\mathrm{t}$. If the MBR of the triangle $t$ does not include the point $p$, the triangle $t$ does not include the point $p$. The time which is taken to create the Delaunay Triangulation is ten times less than the traditional method by using this method to estimate which triangle includes the inserting point $p$.

As shown in Fig. (2), the black points in (a) and (b) represent different interpolation points $I_{1}$ and $I_{2}$. Find triangles of black interpolation points, and red points of every triangle are first order neighboring, which is marked as $N_{1}$, as shown in (a) and (b). In (a), the number of interpolation point $I_{1}$ 's first order neighboring is 7 . In (b), the number of interpolation point $I_{2}$ 's first order neighboring is 6. Different interpolation point has different number of reference points. The same way, the first order neighboring of the red points are interpolation points' second order neighboring, which is marked as $N_{2}$, as shown in (a) and (b). In (a), the number of interpolation point $I_{1}$ 's second order neighboring is 14 .

In (b), the number of interpolation point $I_{2}$ 's second order neighboring is 9. Take first order neighboring and second order neighboring as reference points to local RBF interpolation. In the whole process of interpolation, there's no need to set any parameter, the reference points are completely adaptively chosen. The adaptive search strategy of this paper can make the reference points evenly distributed around the interpolation points.

The algorithm of adaptively choosing first and second order neighboring is shown below.
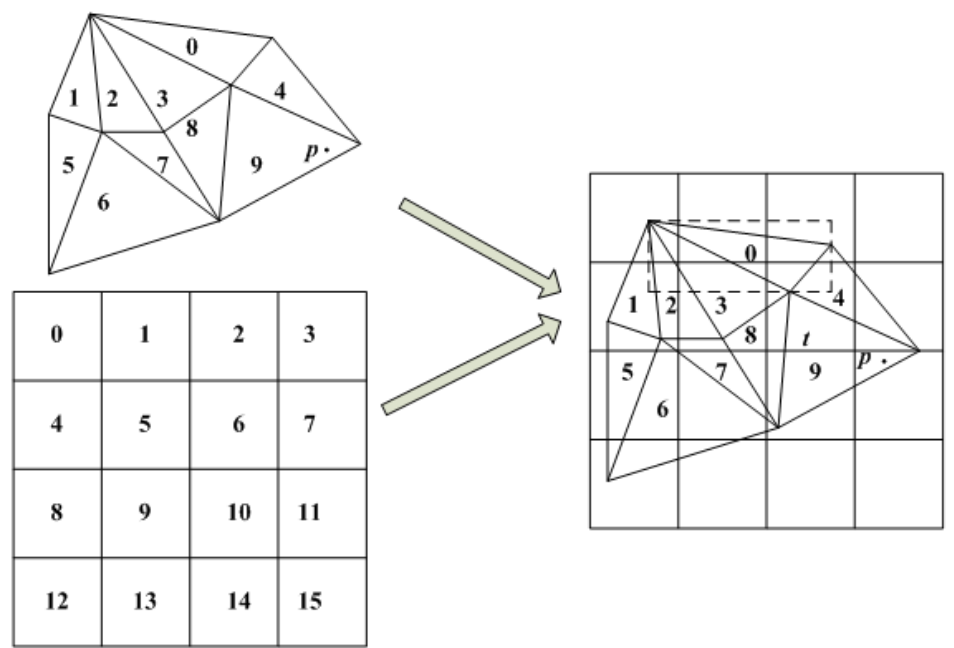

\begin{tabular}{|c|c|c|c|}
\hline $\begin{array}{c}0,1,2, \\
3\end{array}$ & $0,2,3$ & $0,3,4$ & 4 \\
\hline $\begin{array}{c}0,1,2,3 \\
5,6,7\end{array}$ & $\begin{array}{c}0,2,3,6 \\
7,8\end{array}$ & $\begin{array}{c}0,3,4,7 \\
8,9\end{array}$ & 4,9 \\
\hline $5,6,7$ & $6,7,8$ & $\begin{array}{c}6,7,8, \\
9\end{array}$ & 9 \\
\hline 5,6 & 6 & & \\
\hline
\end{tabular}

Fig. (1). The regular grid index. 


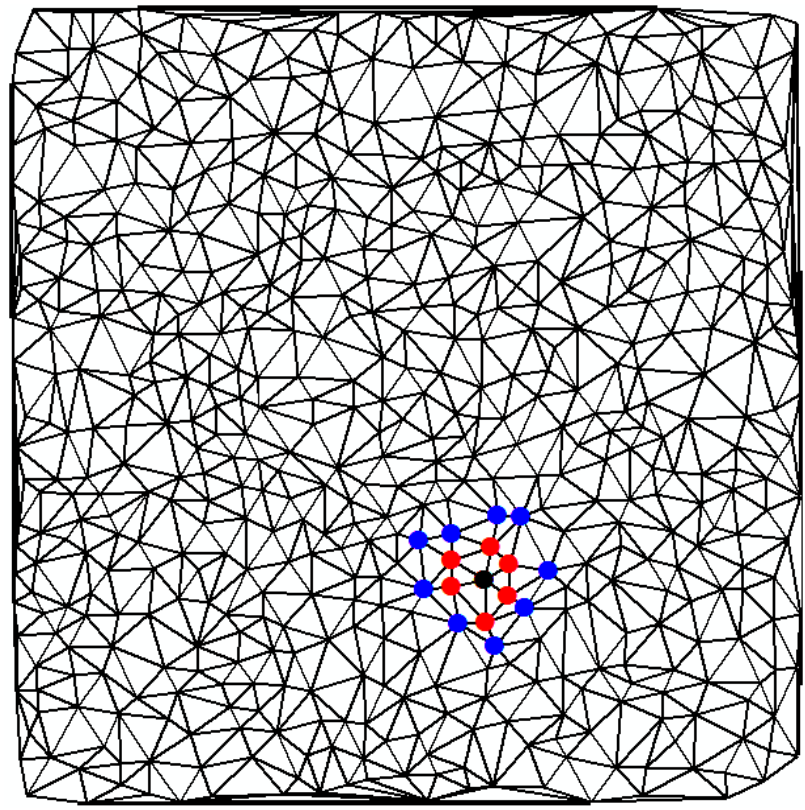

(a) Interpolation points $I_{1}$ and its reference points

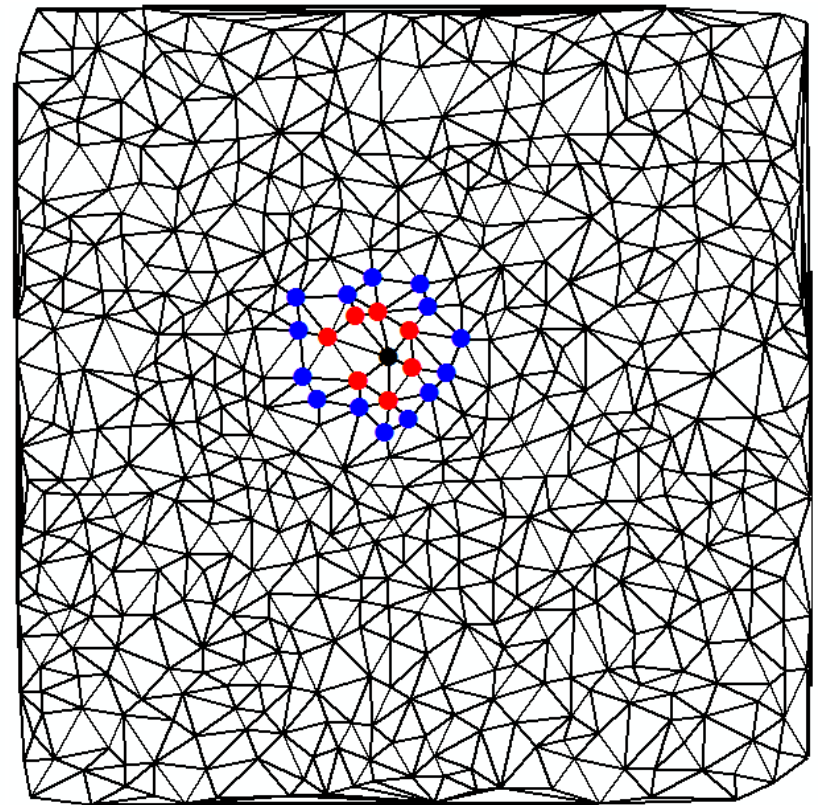

(b) Interpolation points $I_{2}$ and its reference points

Fig. (2). Natural neighbor selections of the first and second order.

Step1: Construct $\mathrm{DT}_{S}$ based on original sample point set $S$.

Step2: Equidistantly subdivide the $S$, and take the grid's nodes as interpolation points set $I=\left\{I_{1}, I_{2}, \ldots, I_{n}\right\}$.

Setp3: Insert the interpolation point $I_{i}$ into $\mathrm{DT}_{S}$, and rebuild the local optimum $\mathrm{DT}_{S}$.

Step4: Search the triangles that contains the interpolation points in the new $\mathrm{DT}_{S}$, the points without repetitive ones are the first order natural neighboring.

Step5: Repeat step3 to the results of step4 to search the second order natural neighboring.

Chose first and second order natural neighboring of interpolation points as reference points according to the algorithm above. The details of RBF interpolation method are shown below.

\subsection{RBF interpolation}

The original sample point set is $\left\{\mathbf{u}_{j}, f\left(\mathbf{u}_{j}\right)\right\}_{j=1}^{N}$, the interpolation formula can be expressed as:

$f^{*}(\mathbf{u})=\sum_{j=1}^{N} \lambda_{j} \varphi\left(\left\|\mathbf{u}-\mathbf{u}_{j}\right\|_{2}\right), \quad \mathbf{u} \in R^{d}$

The $\mathbf{u}$ represents coordinates of any interpolation point, $\mathrm{f}\left(\mathbf{u}_{j}\right)$ represents the height of any sampling point, $\varphi\left(\left\|\mathbf{u}-\mathbf{u}_{j}\right\|_{2}\right)$ is radial basis function, $\mathbf{u}_{j}$ represents coordinates of sampling points, $\left\|\mathbf{u}-\mathbf{u}_{j}\right\|_{2}$ is the Euclidean distance between sampling point and any interpolation points, $\lambda_{j}$ is the set of coefficient's linear combination. All these variables meet the conditions below.
$\sum_{j=1}^{N} \lambda_{j} \varphi\left(\left\|\mathbf{u}_{k}-\mathbf{u}_{j}\right\|_{2}\right)=f\left(\mathbf{u}_{k}\right), \quad \forall k$

To confirm interpolation model, the coefficient $\lambda_{j}$ need to be resolved. There are $N$ functions in (2), whose matrix forms are:

$\boldsymbol{\Phi}\left[\begin{array}{llll}\lambda_{1} & \lambda_{2} & \cdots & \lambda_{N}\end{array}\right]^{\mathrm{T}}=\left[\begin{array}{llll}f_{1} & f_{2} & \cdots & f_{N}\end{array}\right]^{\mathrm{T}}$

Among them,

$\boldsymbol{\Phi}=\left(\begin{array}{cccc}\varphi\left(\left\|\mathbf{u}_{1}-\mathbf{u}_{1}\right\|_{2}\right) & \varphi\left(\left\|\mathbf{u}_{1}-\mathbf{u}_{2}\right\|_{2}\right) & \cdots & \varphi\left(\left\|\mathbf{u}_{1}-\mathbf{u}_{N}\right\|_{2}\right) \\ \varphi\left(\left\|\mathbf{u}_{2}-\mathbf{u}_{1}\right\|_{2}\right) & \varphi\left(\left\|\mathbf{u}_{2}-\mathbf{u}_{2}\right\|_{2}\right) & \cdots & \varphi\left(\left\|\mathbf{u}_{2}-\mathbf{u}_{N}\right\|_{2}\right) \\ \vdots & \vdots & \ddots & \vdots \\ \varphi\left(\left\|\mathbf{u}_{N}-\mathbf{u}_{1}\right\|_{2}\right) & \varphi\left(\left\|\mathbf{u}_{N}-\mathbf{u}_{2}\right\|_{2}\right) & \cdots & \varphi\left(\left\|\mathbf{u}_{N}-\mathbf{u}_{N}\right\|_{2}\right)\end{array}\right)_{N \times N}$

Resolve (3) to get coefficient set,

$\left[\begin{array}{llll}\lambda_{1} & \lambda_{2} & \cdots & \lambda_{n}\end{array}\right]^{\mathrm{T}}=\boldsymbol{\Phi}^{-1}\left[\begin{array}{llll}f_{1} & f_{2} & \cdots & f_{n}\end{array}\right]^{\mathrm{T}}$

Plug (4) into (1) to get the expression of RBF, which is the interpolation mode $[13,14]$.

\section{EXPERIMENTAL ANALYSIS}

The original DEM is from website (http://gdex.cr.usgs.gov/gdex/), as shown in Fig. (3). Choose three different landform types to conduct the experiments of ALRBF interpolation. The range of these three DEMs is $10 \mathrm{~km} * 10 \mathrm{~km}$, and the resolution of them is $30 \mathrm{~m} * 30 \mathrm{~m}$. The intervals of elevation are respectively [48,376], [524, 1534] and [4248, 5230]. Randomly select 5000 points of each DEM as sampling points. 


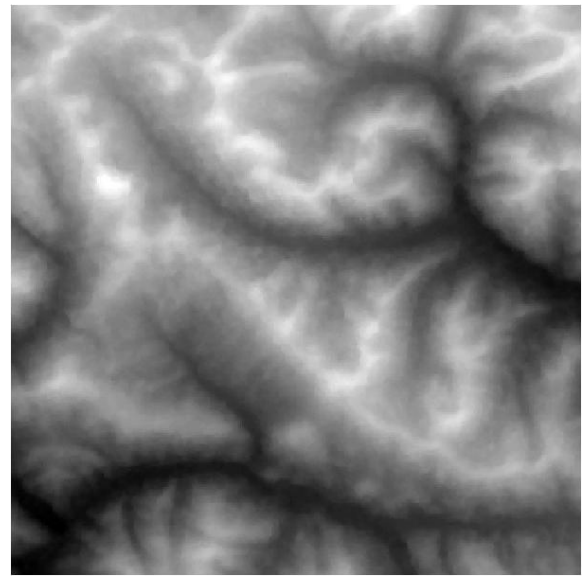

(a)

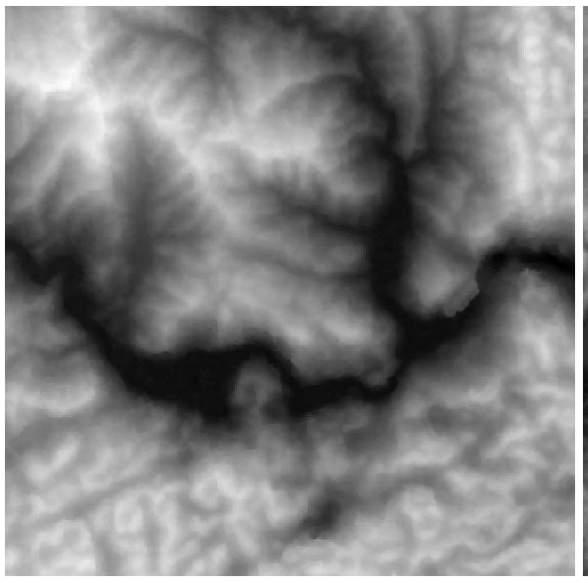

(b)

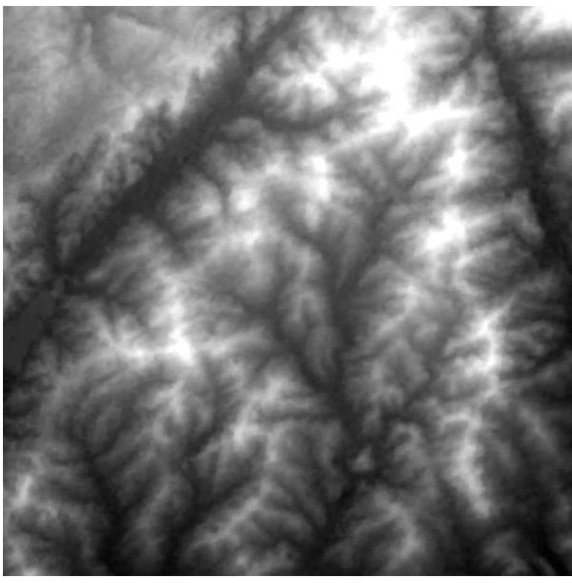

(c)

Fig. (3). DEMs of different landform types.

Equidistantly subdivide the original DEM to get about 62500 interpolation points. To estimate the accuracy of ALRBF interpolation, the correlative errors are shown below [15].

Definition 3-1 (Error) suppose that the true value of the elevation data is denoted by $Z$ and the observed value is denoted by $z$. The error is expressed by the following expression $\varepsilon=Z-z$.

Definition 3-2 (Max Error) The Max Error is expressed by the following expression $\varepsilon_{\max }=\max \left\{\varepsilon_{i}\right\}$.

Definition 3-3 (Min Error) The Min Error is expressed by the following expression $\varepsilon_{\min }=\min \left\{\varepsilon_{i}\right\}$.
Definition 3-4 (Mean Error) The Mean Error is expressed by the following expression $\varepsilon_{\mathrm{ME}}=\sum_{i=0}^{62500} \varepsilon_{i} / 62500$

Definition 3-5 (Root-Mean-Square Error) The RootMean-Square Error is expressed by the following expression $\varepsilon_{\mathrm{RMSE}}=\sqrt{\sum_{i=0}^{62500} \varepsilon_{i}^{2} / 62500}$

Calculate $\varepsilon_{\max }, \varepsilon_{\min }, \quad \varepsilon_{\mathrm{ME}}$ and $\varepsilon_{\mathrm{RMSE}}$ of every interpolation point. The results are shown in Table 1. The study region (a) has the smallest interpolation error, because the extent of the break slope of (a) is quite smaller than the others.

Table 1. Error statistics(unit: $\mathbf{m}$ ).

\begin{tabular}{|c|c|c|c|c|}
\hline Landform types & $\varepsilon_{\max }$ & $\varepsilon_{\min }$ & $\varepsilon_{\mathrm{ME}}$ & $\mathcal{E}_{\mathrm{RMSE}}$ \\
\hline \hline $\mathrm{a}$ & 53.592 & -52.218 & 1.314 & 8.235 \\
\hline $\mathrm{b}$ & 141.583 & -115.181 & 9.455 & 22.191 \\
\hline $\mathrm{c}$ & 1581.473 & -591.720 & -5.341 & 34.607 \\
\hline
\end{tabular}

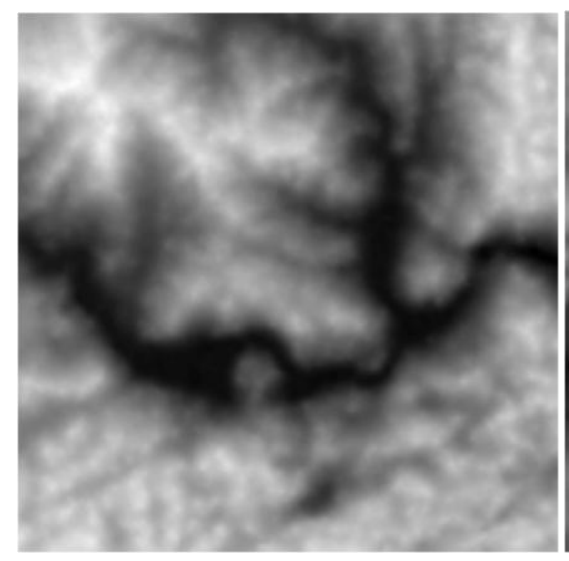

(a)

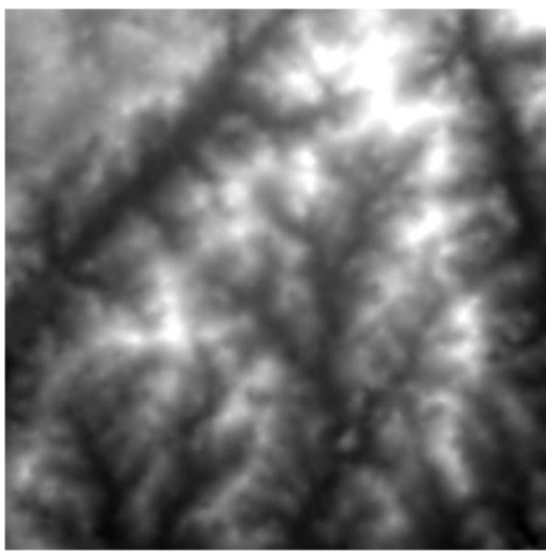

(b)

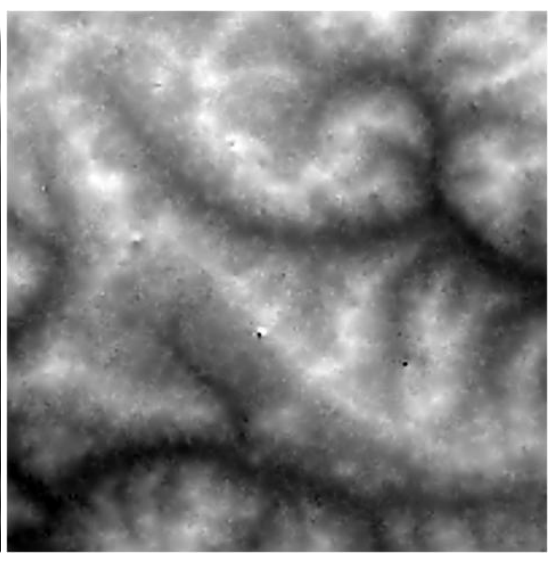

(c)

Fig. (4). Interpolation results of three regions. 
The interpolation results of three regions are shown in Fig. (4). Generally speaking, the interpolation results are familiar with the original DEMs, which represents that ALRBF interpolation can reconstruct the trend of terrain. The noise points in (c) are affected by the local over-fitting, which is radically caused by the huge break of terrain. There are no noise points in (a) and (b), because extents of the terrain break of them are smaller than (c).

\section{CONCLUSION}

By introducing the flaws of the way choosing reference points of RBF interpolation, represent the definition and advantages of spatial relationship of natural neighbor, based on which the local RBF with spatial relationship of natural neighbor interpolation method was proposed in this paper. The whole process of ALRBF contains construction of DTs, searching first and second order neighboring and RBF interpolation. Among them, the step searching first and second order neighboring is most time-consuming. So, spatial index and local update is used in this paper to raise the speed.

Conducting experiments based on three DEMs with different landform types, the feasibility of ALRBF is tested through analysis. The advantages of ALRBF are: (1) no need to set any parameter. The reference points can be chosen adaptively; (2) the problem of huge computations is avoided by choosing first and second order neighboring as reference points.

\section{CONFLICT OF INTEREST}

The authors confirm that this article content has no conflict of interest.

\section{ACKNOWLEDGEMENTS}

This work has been supported by the National Natural Science Foundation of China (No.41271383) and by the foundation of Inovative research program of Jiangsu province of China (No.CXLX13_376).

\section{REFERENCES}

[1] S. B. Kim and T. G. Kim, "Intelligent interpolation methods for automatic generation of an accurate digital elevation model," U. S. Patent 6,748,121, June 8, 2004.

[2] X. Ren, J. J. Liu, C. L. Li, L. L. Mou, and X. D Zou, "Method for manufacturing digital elevation model by using data of laser altimeter," C. N. Patent 101,950,436, Jan. 19, 2011.

[3] M. C. Shi, C. B. Sun, G. Cao, T. H. LI, S. Li and Z.W. Huang, "Method and device for generating digital elevation model from contour map," C. N. Patent 102,339,478, Jan. 1, 2012.

[4] Y. X. Liu, W. Zhang, M. C. Li, Z. Li, K. Yang, L. Cheng, L. H. Tong, W. Hu, W. T. Cai, and Y. M. Chen, "Medium-resolution remote sensing image discrete point DEM construction method based on medium value filtering," C.N. Patent 102436679, May 2, 2012.

[5] N. A. Van, "Digital elevation model," W. O. Patent 2,013,121,340, Aug. 22, 2013.

[6] G. A. Tang, X. J. Liu, and G. N. Lv, Digital Elevation Model and Principles Methodology in Geographical Analysis. Beijing: Science Press, 2005.(In Chinese).

[7] R. Franke, "Scattered data interpolation: tests of some methods", Math. Comp, vol. 38(157), pp.181-200, Jan. 1982.

[8] M. F. Cohen, C. F. Rose III, and P. P. J Sloan, "Interpolation using radial basis functions with application to inverse kinematics," U. S Patent 7,024,279, Apr. 4, 2006.

[9] S. H. Chen, B. Gao, and J.H. Wu, "Method and apparatus for predicting petrophysical properties from $\mathrm{nmr}$ data in carbonate rocks," W. O. Patent, 2,012,037,317, May 31, 2012.

[10] R. K. Beatson, W. A. Light, and S. Billings, "Fast solution of the radial basis function interpolation equations: domain decomposition methods", SIAM J. Sci. Comput., Vol. 22(5), pp.1717-1740, December, 2001.

[11] R. Yokota, L. A. Barba, and M. G. Knepley, "PetRBF-A parallel $\mathrm{o}(\mathrm{n})$ algorithm for radial basis function interpolation with gaussians", Comput. Methods Appl. Mech. Engrg., Vol.199(25), pp.1793-1804, June, 2010.

[12] P. Duan, J.T. Li, X. Q. Zuo, and J. L, "A new interpolation model of convex hull in Delaunay triangulation", International Symposium on Spatial Analysis, Spatial-Temporal Data Modeling, and Data Mining, Wuhan, China, 2009, pp.74924S1-9.

[13] M. D. Buhmann, Radial basis functions: theory and implementtations. Cambridge: Cambridge university press, 2003.

[14] H. Wendland, Scattered data approximation. Cambridge: Cambridge University press, 2005.

[15] J. Li, A review of spatial interpolation methods for environmental scientists. Canberra: Geoscience Australia press, 2008.

Received: May 22, 2014

Revised: July 31, 2014

Accepted: August 04, 2014

(c) Duan et al.; Licensee Bentham Open.

This is an open access article licensed under the terms of the Creative Commons Attribution Non-Commercial License (http://creativecommons.org/licenses/by-nc/3.0/) which permits unrestricted, non-commercial use, distribution and reproduction in any medium, provided the work is properly cited. 